\title{
The acidic, glutamine-rich Mpn474 protein of Mycoplasma pneumoniae is surface exposed and covers the complete cell
}

\section{Correspondence \\ Jan Hegermann \\ jhegerm1@gwdg.de}

Received 18 September 2007

Revised 9 January 2008

Accepted 15 January 2008

\author{
Jan Hegermann, ${ }^{1} \dagger$ Sven Halbedel, ${ }^{1}$ Roger Dumke, ${ }^{2}$ Jörg Regula, ${ }^{3} \ddagger$ \\ Razif R. Gabdoulline, ${ }^{4}$ Frank Mayer, ${ }^{1}$ Jörg Stülke ${ }^{1}$ and Richard Herrmann ${ }^{3}$ \\ ${ }^{1}$ Abteilung für Allgemeine Mikrobiologie, Institut für Mikrobiologie und Genetik, Grisebachstr. 8, \\ D-37077 Göttingen, Germany \\ ${ }^{2}$ Institut für Medizinische Mikrobiologie und Hygiene, Technische Universität, \\ Medizinische Fakultät Carl Gustav Carus, Fetscherstr. 74, D-01307 Dresden, Germany \\ ${ }^{3}$ Zentrum für Molekulare Biologie der Universität Heidelberg (ZMBH), Im Neuenheimer Feld 282, \\ D-69120 Heidelberg, Germany \\ ${ }^{4}$ Molecular and Cellular Modeling Group, EML Research, Schloss-Wolfsbrunnenweg 33, \\ D-69118 Heidelberg, Germany
}

The protein Mpn474 encoded by the mpn474 gene of the human-pathogenic Mycoplasma pneumoniae contains 1033 amino acids and has an isoelectric point of 4.79 , which is caused by the large excess of glutamic acid residues (11\%). Although the protein lacks recognizable export signals we showed by immuno-electron microscopy that Mpn474 is surface exposed, covering the cell completely. By combining cross-linking and careful treatment of the bacterial cells with Triton X-100, we found that this protein is weakly bound to the cell surface, while the true transmembrane protein Mpn141 (adhesin P1) is firmly attached under the same experimental conditions. A transposon mutant in the mpn474 gene, which has no obvious phenotype, served as negative control for the immunodetection.

\section{INTRODUCTION}

Mycoplasma pneumoniae is one of the smallest known selfreplicating bacteria, with a genome size of $816.394 \mathrm{bp}$ and 688 protein-encoding genes (Himmelreich et al., 1996; Dandekar et al., 2000). It has a parasitic lifestyle and causes in its only natural host, humans, infections of the respiratory tract with possible extrapulmonary complications (Waites \& Talkington, 2004).

The phylogenetically closest relative of $M$. pneumoniae is Mycoplasma genitalium, with a genome size of only about $580 \mathrm{~kb}$ (Fraser et al., 1995). Despite the complete lack of a rigid cell wall, both $M$. pneumoniae and $M$. genitalium exhibit a very specific asymmetric cell shape characterized by a polar extension named the 'tip'. Its correct formation is essential for adherence to the host cell's receptors and for motility (Krause \& Balish, 2004). Inside the tip, complex protein structures have been observed (Biberfeld \&

†Present address: European Neuroscience Institute, Grisebachstr. 5, 37077 Göttingen, Germany.

‡Present address: Roche Diagnostics GmbH, Nonnenwald 2, 82377 Penzberg, Germany.

Supplementary material is available with the online version of this paper.
Biberfeld, 1970; Meng \& Pfister, 1980; Göbel et al., 1981; Regula et al., 2001; Hegermann et al., 2002; Henderson \& Jensen, 2006; Seybert et al., 2006) which are thought to be part of a cytoskeleton-like structure. By analogy with eukaryotic cells, such structures could provide the framework for maintaining and stabilizing the asymmetric cell shape.

Due to the lack of a rigid cell wall, M. pneumoniae cells are disrupted when treated with aqueous solutions of Triton $\mathrm{X}-100$. In the course of proteome analyses, M. pneumoniae proteins were classified by their solubility in this detergent. So far, all proteins which are components of a cytoskeleton-like structure are part of the Triton X-100-insoluble fraction (Regula et al., 2001). In addition, about 40 more proteins belonging to different functional categories such as energy metabolism, translation/transcription, heat shock, chaperones and unknown functions have also been found in this fraction. Among the proteins with unknown function was Mpn474 (previously designated P01_orf1033 (Himmelreich et al., 1996)). This large protein, consisting of 1033 amino acids, attracted our attention because it has predicted extended coiled-coil structures which are characteristic for several proteins of the putative mycoplasma cytoskeleton and, based on sequence similarities, Mpn474 
was grouped in a family of paralogous proteins (Himmelreich, 1997) with the proteins encoded by Mpn310 (HMW2), Mpn387 and Mpn426 (P115). HMW2 plays an essential role in tip formation (Balish \& Krause, 2003). P115 belongs to the SMC (structural maintenance of chromosomes) proteins, which are involved in organization and division of bacterial chromosomes (Hirano, 2005), and Mpn387 has recently been associated with the gliding motility of $M$. pneumoniae (Hasselbring et al., 2006). Further, a recent study showed that at least a certain percentage of Mpn474 is phosphorylated like several other proteins involved in formation of a cytoskeleton-like structure and cytadherence ( $\mathrm{Su}$ et al., 2007). The sum of these data prompted us to do a functional analysis of Mpn474. It was the aim of this study, as a first step towards a functional analysis, to localize the protein Mpn474 in M. pneumoniae cells, using a knockout mutant of the mpn474 gene as negative control. We took advantage of the observation that the cytoplasmic membrane can be removed from chemically fixed cells by aqueous solutions of Triton X-100, while the cells maintain their shape (Hegermann et al., 2002). This approach allows immunocytological analyses in dependence on the presence or absence of the cytoplasmic membrane.

\section{METHODS}

Bacterial strains and growth conditions. The strains used in this study were M. pneumoniae M129 (ATCC 29342) in the 33rd broth passage, and its isogenic mutant derivative GPM70 (mpn474:: mini$\left.\mathrm{Tn}, \mathrm{Gm}^{\mathrm{R}}\right)$. M. pneumoniae was grown at $37^{\circ} \mathrm{C}$ in $150 \mathrm{~cm}^{2}$ tissueculture flasks containing $100 \mathrm{ml}$ modified Hayflick medium as described previously (Regula et al., 2000). Strains harbouring transposon insertions were cultivated in the presence of $80 \mu \mathrm{g}$ gentamicin $\mathrm{ml}^{-1}$. Agar plates were prepared by adding $1 \%$ agar to the Hayflick medium.

Synthesis of recombinant Mpn474 and preparation of antiserum against Mpn474. The cosmid pcosMPP01 (Wenzel \& Herrmann, 1989) was used as a template for amplifying the $m p n 474$ gene by PCR using the primer pair P01_orf1033-start $\left(5^{\prime}\right.$ CATGCCATGGAGTTTTTAGAACAAGAAGG-3') and P01_orf1033BglII (5'-GATAGATCCTGCTTGCCATTGATCTTAGCTA- $\left.{ }^{\prime}\right)$. The PCR product was digested with BglII and cloned into the vector pQE60 (Qiagen), which was first cut with NcoI, made blunt with phage T4 DNA polymerase and subsequently digested with BglII. This construct allowed the regulated expression of the complete Mpn474 with eight additional amino acids at the C-terminal end (Arg, Ser and $6 \times$ His) in E. coli SG13009 (Qiagen). Since the protein was insoluble in Escherichia coli, purification was done under denaturing conditions. Mpn474 was purified to homogeneity by immobilized metal chelate affinity chromatography, and gel filtration and anionexchange chromatography using the Äkta Explorer 10 System (Pharmacia Amersham). The purified protein was dialysed against PBS (0.15 M NaCl, $10 \mathrm{mM}$ sodium phosphate, $\mathrm{pH} 7.4)$ prior to immunization. Rabbits were immunized by standard procedures with 250-500 $\mu$ g protein per subcutaneous injection (Proft \& Herrmann, 1994). The monospecific Mpn474 antiserum (serum \#41804) could be diluted up to 1:20000 for Western blotting experiments.

Western blot analysis. For Western blot analysis, M. pneumoniae crude cell extracts (Halbedel et al., 2004) were separated on $10 \%$
SDS-polyacrylamide gels. After electrophoresis, the proteins were transferred to a PVDF membrane (Bio-Rad) by electroblotting. Mpn474 was detected with polyclonal antibodies (serum \#41804, dilution 1:10000) raised against recombinant Mpn474 of $M$. pneunomiae. Antibodies were visualized by using anti-rabbit IgG-AP secondary antibodies (Promega) and the $\mathrm{CDP}^{\star}$ detection system (Roche Diagnostics).

Southern blot analysis. Southern blot analysis was done as recently described (Halbedel et al., 2006). Briefly, chromosomal DNA of $M$. pneumoniae was isolated and digested with EcoRV and XhoI. The fragments were separated using $1 \%$ agarose gels, transferred onto a positively charged nylon membrane (Roche Diagnostics) and probed with digoxigenin-labelled riboprobes obtained by in vitro transcription with T7 RNA polymerase (Roche Diagnostics) using PCRgenerated fragments as templates. Primer pairs for the amplification of mpn474 and aac-ahpD gene fragments were SH74 (5'-ACTCCTTCAAACCCAACAAGTC-3')/SH75 (5'-CTAATACGACTCACTATAGGGAGACTTGCAATTGTTGTAACTGCG-3') and SH62/SH63 (Halbedel et al., 2006), respectively. The reverse primers contained a T7 RNA polymerase recognition sequence (underlined in SH75). In vitro RNA labelling, hybridization and signal detection were carried out according to the manufacturer's instructions (DIG RNA labelling kit and detection chemicals; Roche Diagnostics).

Harvesting of $\boldsymbol{M}$. pneumoniae cells. The medium was discarded and the adhering cells were washed three times in the flask with PBS and then scraped off in $2 \mathrm{ml}$ PBS. For microscopy, the cells were disaggregated by pulling them up and down five times in a $5 \mathrm{ml}$ glass pipette. Freshly harvested cells of $M$. pneumoniae were put on ice immediately. Formaldehyde and glutaraldehyde were added to final concentrations of $3.7 \%$ and $0.25 \%$ and the cells were incubated in the fixing solution on ice for at least $30 \mathrm{~min}$. Cells were stored in this solution at $4{ }^{\circ} \mathrm{C}$ until use. Storage was not longer than 10 days.

Electron microscopy. For the observation of whole cells we used the whole-mount procedure as follows. Carbon-coated Formvar-nickel grids were put on drops of fixed cell suspension for $5 \mathrm{~min}$. The grids were washed by floating them three times for $1 \mathrm{~min}$ on drops of PBS. Optionally, the cells were treated with Triton X-100. For this purpose, the grids were incubated for $10 \mathrm{~min}$ on drops of $2 \%$ Triton X-100 in PBS containing $1 \mathrm{M} \mathrm{NaCl}$, and washed again three times on drops of PBS. Subsequently, the samples were negatively stained with $3 \%$ phosphotungstic acid ( $\mathrm{pH} 7$ ) and observed in a Philips EM 301 or a Zeiss EM 902A at $80 \mathrm{kV}$ operated in the bright-field mode.

For immunolocalization on ultrathin sections, fixed cells of $M$. pneumoniae were washed three times in PBS, with centrifugation steps of 2 min at 2000 r.p.m. in an Eppendorf table centrifuge at $4{ }^{\circ} \mathrm{C}$. Subsequently, the cells were either immediately embedded in Lowicryl-K4M resin or treated with Triton X-100 (2\% Triton X100 in PBS containing $1 \mathrm{M} \mathrm{NaCl}, 10 \mathrm{~min}$ ) and washed three times in PBS prior to embedding. For clearer visualization of the membrane, cells were stained with osmium tetroxide prior to embedding in Spurr resin. Embedding and ultrathin sectioning were carried out as described previously (Carlemalm et al., 1982; Hoppert, 2003). Ultrathin sections were mounted on Formvar-nickel grids, optionally immunolabelled and stained either with $3 \%$ phosphotungstic acid ( $\mathrm{pH} 7)$ (Lowicryl sections) or with uranyl acetate and lead citrate (Hoppert, 2003) and observed in a Philips EM 301 or a Zeiss EM $902 \mathrm{~A}$ at $80 \mathrm{kV}$ operated in the bright-field mode.

Immunogold labelling. Grids containing whole cells were put upside down on drops of antibodies directed against Mpn474 (or against Mpn141) for $1 \mathrm{~h}$, washed three times for $5 \mathrm{~min}$ on drops of PBS and then incubated on the secondary antibody (Goat anti-rabbitgold, $10 \mathrm{~nm}$, Sigma) for $30 \mathrm{~min}$, washed again three times in PBS and 
then analysed by electron microscopy. Optionally, grids containing whole cells were incubated for $10 \mathrm{~min}$ on drops of $2 \%$ Triton X-100 in PBS containing $1 \mathrm{M} \mathrm{NaCl}$, and washed again three times on drops of PBS before immunolabelling. Grids containing ultrathin sections were blocked in $2 \%$ BSA in PBS for $1 \mathrm{~h}$, and all further incubation steps were the same as for whole cells, except that the antibodies were diluted in the blocking solution. Washing was done with PBS containing $0.05 \%$ Tween 20. Washing was initiated by applying a jet from a plastic flask to the surface of the grid for $10 \mathrm{~s}$.

\section{RESULTS}

\section{General features of Mpn474}

Based on the annotation of the complete genome sequence the mpn474 gene encodes a 1033 amino acid protein with a high proportion of glutamine (135) and glutamic acid (113) residues. The latter are responsible for the isoelectric point of 4.79, which makes Mpn474 one of the most acidic proteins of $M$. pneumoniae. Mpn474 contains several internal short amino acid repeats (LDQTQLLDELV at positions 192 and 227, HELLQESYNK at 727 and 828, NQVNLEKQ at 882 and 934, QEQLA at 577, 686 and 485, QXQLE at 321, 374, 935, 823 and 314). Their importance is presently unknown. The expression of Mpn474 has been confirmed by $2 \mathrm{D}$ gel electrophoresis combined with mass spectrometry (Regula et al., 2000, 2001) and Western blotting (Regula, 1999). In both analyses, several smaller degradation products were observed in addition to the mature Mpn474. The undegraded Mpn474 has a predicted molecular mass of $118078 \mathrm{Da}$, but it migrates slightly slower in SDS-PAGE, indicating a molecular mass of about $130 \mathrm{kDa}$. However, this is not unprecedented for an acidic protein.

Structure and motif prediction programs showed a high probability of the occurrence of a glutamine-rich region profile, leucine zipper patterns and extended coiled-coil structures. Search for orthologous proteins revealed that the first 300 residues of Mpn474 did not have any significant homology to annotated sequences, while the remaining part has homology to protein $\mathrm{Mg} 328$ from $M$. genitalium, detected as a glutamine-rich low-complexity region and a coiled-coil myosin-tail-like region (residues 355-1033).

Sequence similarity searches in KEGG (Kanehisa et al., 2006) identified region $394-710$ as related to a coiled-coil intermediate filament protein and region $812-932$ as related to membrane-associated flagella accessory protein C (FlacC) families of pfam (Finn et al., 2006).

Mpn474 has neither a signal peptide nor a proposed transmembrane segment, but it can be predicted as an extracellular protein, based on the similarity to bacterial non-classically secreted proteins in terms of various sequence descriptors, such as amino acid composition, secondary structure and disordered regions (Bendtsen et al., 2005).

\section{Isolation of a $M$. pneumoniae mpn474 mutant}

To isolate a $M$. pneumoniae mutant strain devoid of a functional mpn474 gene we applied the 'haystack mutagenesis' strategy as outlined previously (Halbedel et al., 2006). The strategy is based on an ordered collection of pooled random transposon insertion mutants that can be screened for junctions between the transposon and the gene of interest due to transposon insertion. The 64 pools were used in a PCR to detect junctions between the $m p n 474$ gene and the mini-transposon using the oligonucleotides SH74 (ATGAGTGAGCTAACTCACAG) and SH29 (ACTCCTTCAACCCAACAAGTC) (Halbedel et al., 2006; see Fig. 1a). From one pool that gave a positive signal, colony PCR with the 50 individual mutants resulted in the identification of one mpn 474 mutant. The presence of the transposon insertion in mpn474 was verified by Southern blot analysis (Fig. 1b). To test whether this strain contained only a unique transposon insertion, we did another Southern blot using a probe specific for the aac-aphD resistance gene present on the mini-transposon. As shown in Fig. 1(b), only a single band hybridizing with this probe was detected; moreover, this fragment had the same size as the EcoRV-XhoI fragment hybridizing to the mpn474 probe (see Fig. 1b). The isolated mpn474 mutant strain was designated GPM70. The position of the transposon insertion in the mpn474 gene of M. pneumoniae GPM70 was determined by DNA sequencing. The $m p n 474$ gene was disrupted between nucleotides 898 and 899 , resulting in a truncated protein of 299 amino acids with one additional amino acid and the following stop codon encoded by the inserted mini-transposon.

\section{Characterization of the mpn474 mutant strain}

The mutant was indistinguishable from wild-type cells as regards growth behaviour, adherence and electron microscopic appearance. To verify the loss of a functional Mpn474 due to the transposon insertion, we performed a Western blot analysis using protein extracts from the wildtype strain and the mpn474 mutant GPM70. As shown in Fig. 2, the antibodies recognized a large protein that migrated somewhat slower than expected for a protein of a molecular mass of $118 \mathrm{kDa}$. This protein was present in the wild-type strain but not in the mutant GPM70. Thus, no functional Mpn474 is present in the mutant strain (Fig. 2).

\section{The Mpn474 protein is located at the surface of $M$. pneumoniae cells}

M. pneumoniae cells bind antibodies (serum \#41804) against the Mpn474 protein all over their surface, as revealed by electron microscopy. The antibodies were evenly distributed on the cell bodies and filamentous extensions of the cells (Fig. 3a). The observed binding of antibodies did not occur if the cytoplasmic membrane was removed by Triton X-100 prior to immunolabelling (Fig. 3b). Postembedding immuno-electron microscopy 

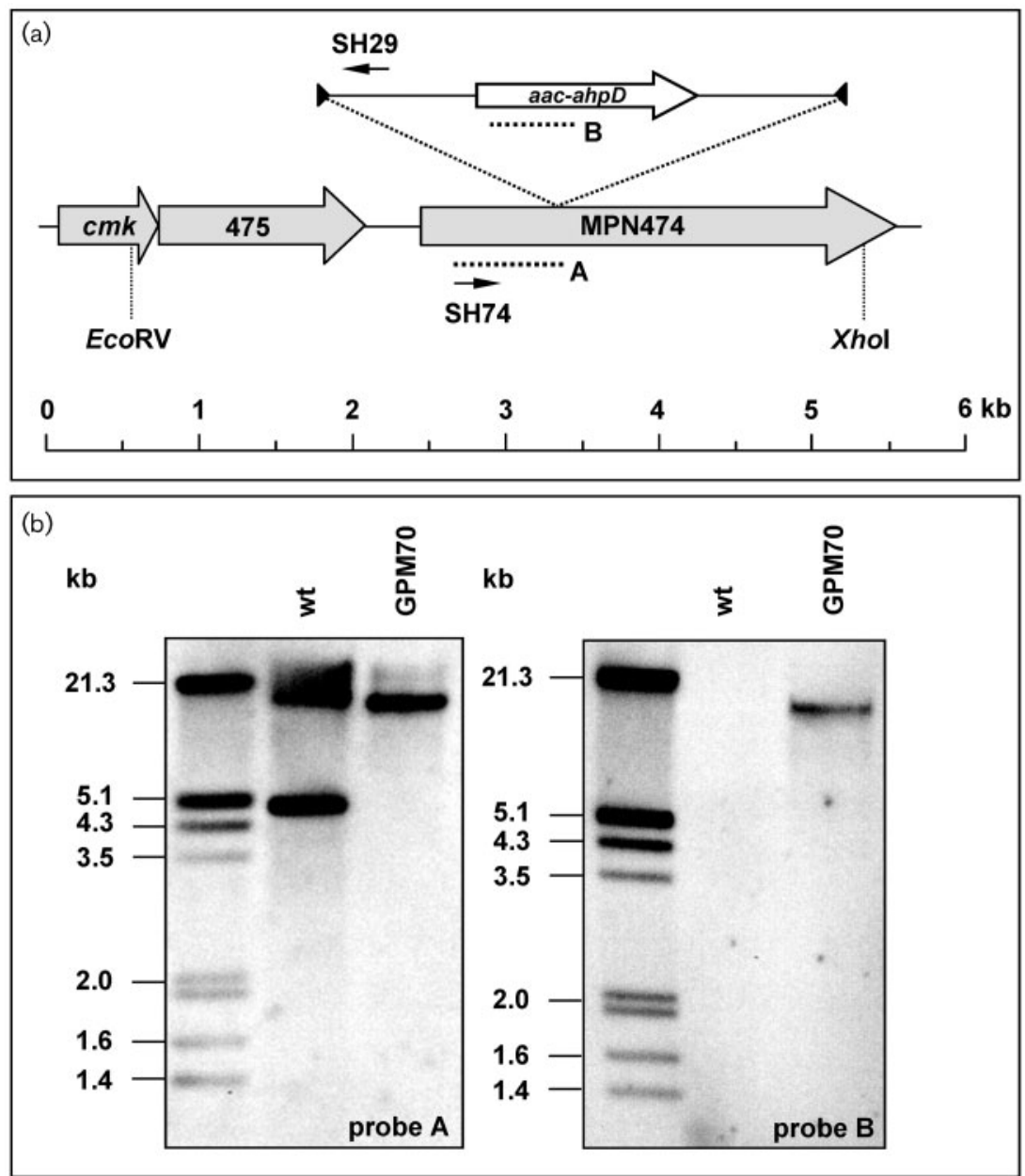

Fig. 1. Isolation of a M. pneumoniae mpn474 transposon insertion mutant. (a) Schematic representation of the genomic region of the mpn474 gene in M. pneumoniae and the site of the transposon insertion in the mpn474 mutant strain GPM70. The annealing sites of the oligonucleotides used for haystack mutagenesis screens and in sequencing reactions to determine the transposon insertion site in GPM70 are indicated by arrows. Probes hybridizing to internal fragments of the $m p n 474$ and the aac-ahpD genes are depicted as dotted lines. (b) Southern blot analysis to confirm the unique insertion of the mini-transposon into the mpn 474 gene of strain GPM70. Chromosomal DNA of the wild-type (wt) and mutant strain (GPM70) was digested using EcoRV and Xhol. Blots were hybridized with the mpn474-specific probe (left) and a probe hybridizing to the aac-ahpD gene of the mini-transposon (right). DIG-labelled DNA molecular mass marker III (Roche Applied Science) served as a standard. revealed that the reaction of the antibodies was restricted to the cell envelope. No reaction was observed in the cytoplasm (Fig. 3, insets 1), independent of the presence

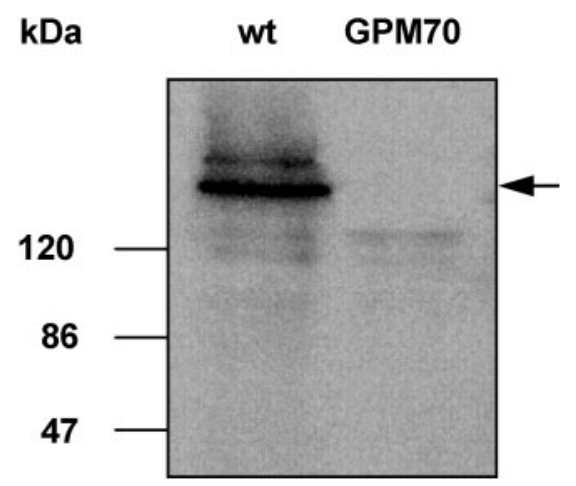

Fig. 2. Western blot analysis of Mpn474 synthesis in $M$. pneumoniae. Antibodies (serum \#41804) raised against $M$. pneumoniae Mpn474 were used to visualize Mpn474 protein levels present in crude cell extracts of $M$. pneumoniae wild-type (wt) and mpn474 mutant GPM70 cells. or absence of the membrane. The effect of membrane removal is best monitored by staining with osmium tetroxide (Fig. 3, insets 2, membrane highlighted by arrowheads), a method that is however not applicable for immunolocalization.

The mpn474-mutant strain GPM70 was used as a control for the specificity of the immunoreaction of the antibodies used. Microscopic and Western-blotting experiments both revealed a slight background reaction within the mutant, which has to be subtracted from the observed reaction in the wild-type. The results of immunogold labelling, comparing the wild-type and the mutant, are shown in Fig. 4.

To show the difference of Mpn474 from a true integral membrane protein, we applied the same experimental procedures to the protein Mpn141 (P1). Mpn141 is the main adhesin of $M$. pneumoniae and was predicted to contain two to five transmembrane segments (depending on the prediction program). Mpn141 is preferentially inserted in the membrane surrounding the tip ( $\mathrm{Hu}$ et al., 1982; Baseman et al., 1982), as confirmed here also by immunogold labelling (See Fig. S1 of the supplementary material, available with the online version of this paper). In 

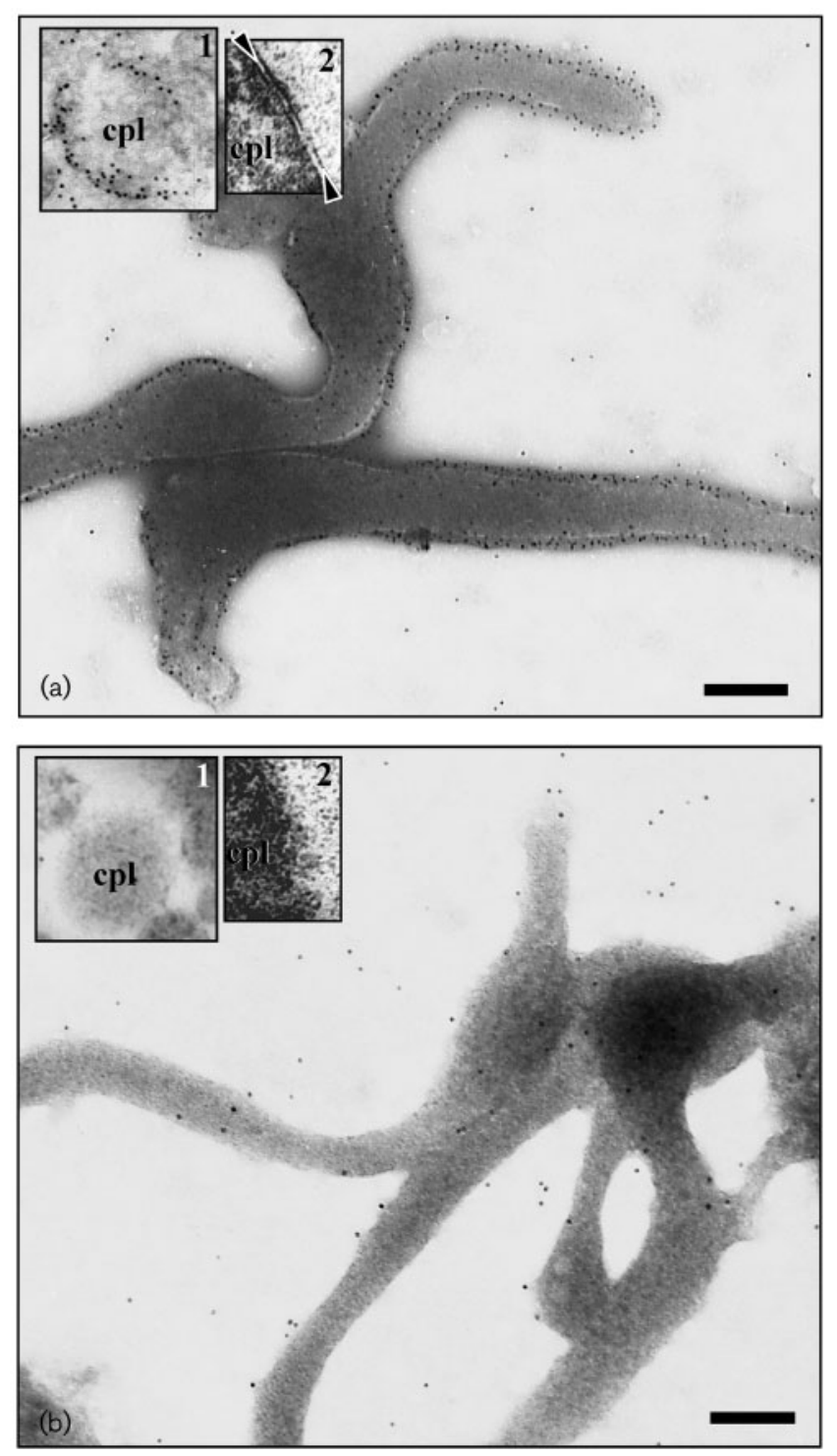

Fig. 3. Immunogold labelling of Mpn474 on M. pneumoniae wildtype cells before and after removal of the membrane. Large images, whole-mount preparation; insets, ultrathin sections prepared for immunolocalization (1) or for good membrane visualization without immunolocalization (2). (a) Intact cells with the cytoplasmic membrane; (b) cells after removal of the membrane. cpl, cytoplasm; arrowheads, cytoplasmic membrane. Bars, $100 \mathrm{~nm}$.

contrast to the Mpn474 protein, Mpn141 is still present on the cell surface after membrane removal, indicating that the two proteins differ in their membrane topology.

\section{Inhibition of growth and attachment to erythrocytes by antiserum to Mpn474}

The surface-exposed localization of Mpn474 suggested that it might be involved in the interaction of M. pneumoniae with its host cell. Therefore, we tested the influence of the monospecific Mpn474 antiserum \#41804 on growth and attachment to sheep erythrocytes. For this purpose, $M$. pneumoniae cells were grown in liquid or solid media in presence of serial dilutions of antiserum \#41804 (Citti et al., 1997 and supplementary material). No significant growth inhibition was observed with this antiserum. An antiserum (\#66708) against the cytoplasmic protein elongation factor G (Mpn227) was used as negative control and an antiserum against whole-cell antigen $(\# 129 \mathrm{M})$ served as a positive control. Both controls reacted as expected with serum \#66708, having no effect on M. pneumoniae growth, while serum \#128M clearly inhibited growth (see supplementary material for experimentation in liquid culture, Fig. S2). Further, the antiserum \#41804 did not inhibit binding of $M$. pneumoniae to sheep erythrocytes in a standard haemadsorption test (Krause et al., 1982) (supplementary material, Fig. S3). These results on growth inhibition and haemadsorption were in accordance with the phenotype of the mpn474 mutant GPM70 as described above 'Characterization of the mpn 474 mutant strain'.

\section{DISCUSSION}

Chemical fixation by glutaraldehyde is achieved by crosslinking of amino acid residues. As the cytoplasmic membrane consists mostly of fatty acids, the effect of glutaraldehyde fixation is weaker here than in the rest of the cell, due to the reduced protein content. Unfixed cells were completely disrupted during lysis in aqueous solutions of Triton X-100, leaving an insoluble fraction which is thought to contain components of a bacterial cytoskeleton (Meng \& Pfister, 1980; Regula et al., 2001; Hegermann et al., 2002). After glutaraldehyde fixation, the cytoplasmic membrane remains soluble and is washed off the cells by TX-100, while the overall shape of the cells is maintained.

Cells devoid of the membrane 'show what they wear below': a structurally organized surface with a helical lining became visible after membrane removal (Hegermann et al., 2002), resembling helical cytoskeletal elements in E. coli and Bacillus subtilis (Carballido-López \& Errington, 2003). The ultrastructure of the mycoplasma cell envelope needs further investigation, as so far it remains a mystery how the cells maintain their asymmetric shape. It seems plausible that a weak, fragile membrane might not be the only layer surrounding the mycoplasma cell.

In ultrathin sections, the membrane appears as a double lining that surrounds the cells; this is not visible after membrane removal (Fig. 3, insets 2). Although the loss of the membrane during the lysis procedure is clearly visible, our data give no information on the possible loss of additional material. The cell envelope might not only consist of the membrane. Previous studies revealed additional material on the outer face of the membrane of mycoplasmas (Bansal et al., 1995; Rosengarten et al., 1988; Neyrolles et al., 1998) and it was postulated that 

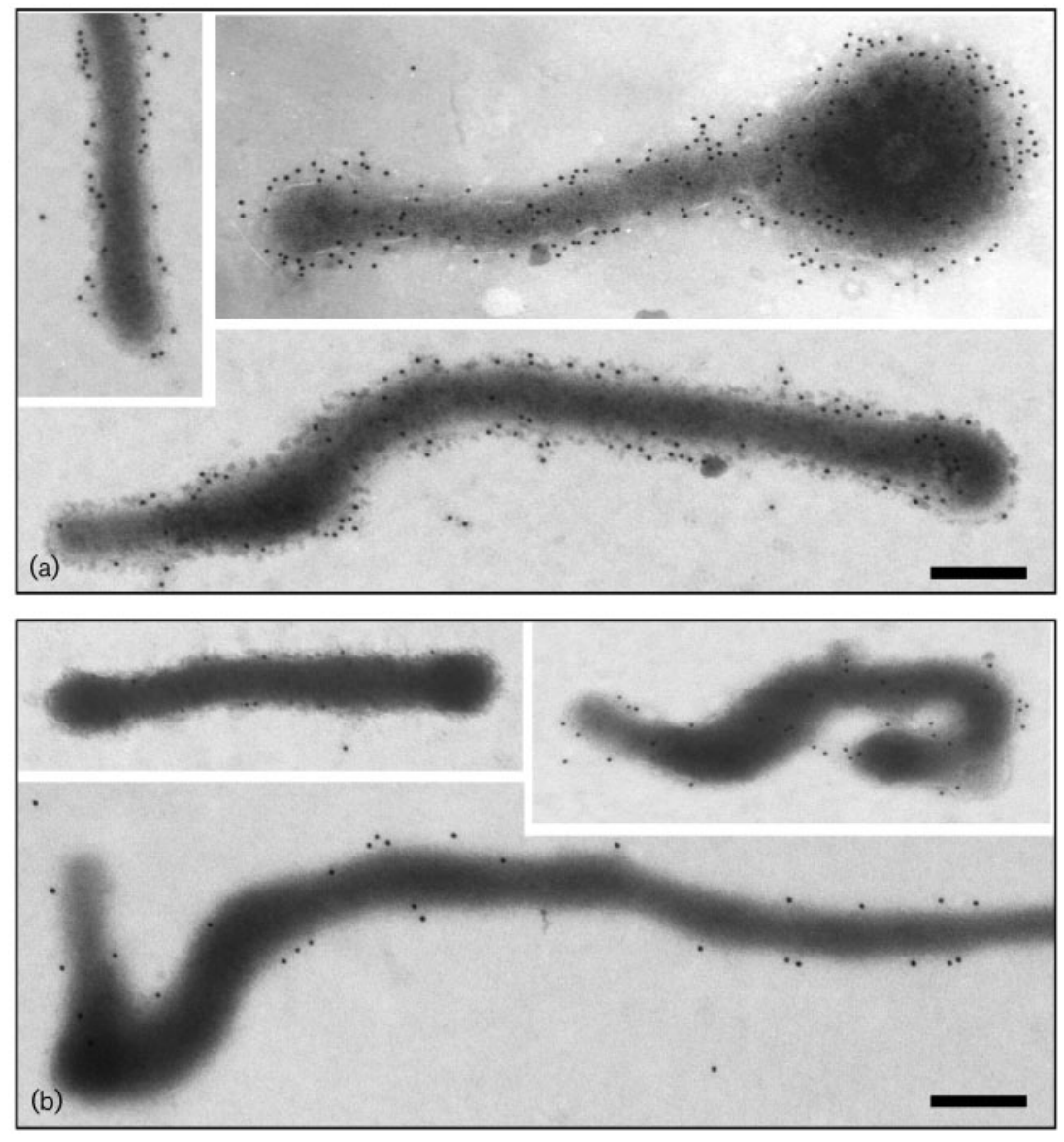

Fig. 4. Immunogold labelling of Mpn474 on M. pneumoniae wild-type (a) and Mpn474 mutant GPM70 (b). Bars, $100 \mathrm{~nm}$. extracellular glycoprotein might be involved in cell adhesion (Wilson \& Collier, 1976).

Antibodies against the Mpn474 protein bind to the whole surface of $M$. pneumoniae cells. To rule out that we might have detected a cytosolic protein that had artificially leaked from the cells due to poor fixation, we also tested the inside of the cells by postembedding staining on thin sections and there was no reaction in the cytosol. Since binding of antibodies to the cell surface is abolished after removal of the membrane, association of Mpn474 with the membrane seems plausible. In contrast to Mpn474, the main adhesin Mpn141 is not detached from the cells by membrane removal under the conditions described. This different behaviour of the two proteins indicates a different way of anchoring to the cell. Mpn474 is apparently not crosslinked to the cell by the bifunctional cross-linker glutaraldehyde, in contrast to Mpn141. A protein without transmembrane regions, that is associated with the outer face of the membrane, will not be cross-linked to proteins of the cell interior, as the glutaraldehyde molecule is smaller than $10 \AA(1 \mathrm{~nm})$ and cannot span the membrane. Therefore, we presume that Mpn474 is not in direct contact with the cell interior, while Mpn141 is. A tight connection to inner cell structures can be expected for Mpn141, as this protein mediates adhesion of the cells to surfaces. Mpn474 is, in contrast, not essential for adhesion, as the mutant strain GPM70 grows adherently to glass and plastic like the wild-type under laboratory conditions (data not shown), but we cannot rule out that wild-type and mutant behave differently in vivo.

The observation that Mpn474 has neither a signal peptide nor a transmembrane segment raises two questions: how is this protein secreted in a signal-peptide-independent pathway and how is it bound to the surface of the cell membrane? The phenomenon that proteins can be secreted without any apparent signal peptide, which occurs in both eukaryotes (Nickel, 2003) and prokaryotes (Bendtsen et al., 2005), has been termed non-classical secretion. Examples of non-classical secretion have been found in Grampositive and Gram-negative bacteria (Bendtsen et al., 2005), but the mechanism and structural components for their export routes are still unknown. Our data reveal that the Mpn474 protein is associated with the cytoplasmic membrane. It might be stabilized there by interacting with membrane proteins with surface-exposed regions or by as yet uncharacterized components of the outer cell surface. To isolate protein complexes containing Mpn474 and one or more binding partners under native conditions is rather difficult, because Mpn474 and membrane proteins, as the most likely interacting components, are only poorly 
soluble. For instance, the tandem affinity purification (TAP) method (Puig et al., 2001), which works well for soluble complexes (Gavin et al., 2002), cannot be applied for this reason. Therefore, theoretical approaches, like searching in the 'database for interacting proteins' (DIP; Salwinski et al., 2004) for interacting protein pairs from other organisms with one of the proteins in the pair being homologous to Mpn474 and another being homologous to any other $M$. pneumoniae protein, seem to be an alternative. Homology-based interference of possible interactions can hardly be considered as reliable prediction (Aloy \& Russell, 2006). Based on the results of such studies, specific experiments could then be done to verify or disprove the interaction of the suggested proteins. We think Mpn474 is a good candidate to study non-classical secretion and to identify proteins which mediate binding of the secreted protein to the cell surface, because $M$. pneumoniae is a relatively simple organism concerning proteome complexity, and the bacterium is surrounded by one membrane only, excluding complications caused by a second membrane or a cell wall.

\section{ACKNOWLEDGEMENTS}

We are grateful to Elsbeth Pirkl and Julia Busse for excellent technical assistance. Rebecca C. Wade and Ake Wieslander are acknowledged for helpful discussions. This work was supported by grants from the Deutsche Forschungsgemeinschaft, the Fonds der Chemischen Industrie (R. H. and J.S.) and from the German Federal Ministry of Education and Research (BMBF Project Bioregio 12212, R. R. G.).

\section{REFERENCES}

Aloy, P. \& Russell, R. B. (2006). Structural systems biology: modelling protein interactions. Nat Rev Mol Cell Biol 7, 188-197.

Balish, M. F. \& Krause, D. C. (2003). Cytadherence and the cytoskeleton. In Molecular Biology and Pathogenicity of Mycoplasmas, pp. 491-518. Edited by S. Razin \& R. Herrmann. New York: Kluver Academic/Plenum Publishers.

Bansal, P., Adegboye, D. S. \& Rosenbusch, R. F. (1995). Immune responses to the capsular polysaccharide of Mycoplasma dispar in calves and mice. Comp Immunol Microbiol Infect Dis 18, 259-268.

Baseman, J. B., Cole, R. M., Krause, D. C. \& Leith, D. K. (1982). Molecular basis for cytadsorption of Mycoplasma pneumoniae. J Bacteriol 151, 1514-1522.

Bendtsen, J. D., Kiemer, L., Fausbol, A. \& Brunak, S. (2005). Nonclassical protein secretion in bacteria. BMC Microbiol 5, 58.

Biberfeld, G. \& Biberfeld, P. (1970). Ultrastructural features of Mycoplasma pneumoniae. J Bacteriol 102, 855-861.

Carballido-López, R. \& Errington, J. (2003). A dynamic bacterial cytoskeleton. Trends Cell Biol 13, 577-583.

Carlemalm, E., Garavito, R. M. \& Villinger, W. (1982). Resin development for electron microscopy and an analysis of embedding at low temperature. J Bacteriol 159, 138-144.

Citti, C., Kim, M. F. \& Wise, K. S. (1997). Elongated versions of Vlp surface lipoproteins protect Mycoplasma hyorhinis escape variants from growth-inhibiting host antibodies. Infect Immun 65, $1773-1785$.
Dandekar, T., Huynen, M., Regula, J. T., Ueberle, B., Zimmermann, C. U., Andrade, M. A., Doerks, T., Sanchez-Pulido, L., Snel, B. \& other authors (2000). Re-annotating the Mycoplasma pneumoniae genome sequence: adding value, function and reading frames. Nucleic Acids Res 28, 3278-3288.

Finn, R. D., Mistry, J., Schuster-Bockler, B., Griffiths-Jones, S., Hollich, V., Lassmann, T., Moxon, S., Marshall, M., Khanna, A. \& other authors (2006). Pfam: clans, web tools and services. Nucleic Acids Res 34, D247-D251.

Fraser, C. M., Gocayane, J. D., White, O., Adams, M. D., Clayton, R. A., Fleischmann, R. D., Bult, C. J., Kerlavage, A. R., Sutton, G. \& other authors (1995). The minimal gene complement of Mycoplasma genitalium. Science 270, 397-403.

Gavin, A. C., Bosche, M., Krause, R., Grandi, P., Marzioch, M., Bauer, A., Schultz, J., Rick, J. M,, Michon, A. M. \& other authors (2002). Functional organization of the yeast proteome by systematic analysis of protein complexes. Nature 415, 141-147.

Göbel, U., Speth, V. \& Bredt, W. (1981). Filamentous structures in adherent Mycoplasma pneumoniae cells treated with nonionic detergents. J Cell Biol 91, 537-543.

Halbedel, S., Hames, C. \& Stülke, J. (2004). In vivo activity of enzymatic and regulatory components of the phosphoenolpyruvate: sugar phosphotransferase system in Mycoplasma pneumoniae. J Bacteriol 186, 7936-7943.

Halbedel, S., Busse, J., Schmidl, S. \& Stülke, J. (2006). Regulatory protein phosphorylation in Mycoplasma pneumoniae: A PP2C-type phosphatase serves to dephosphorylate $\operatorname{HPr}($ Ser-P). J Biol Chem 281, 26253-26259.

Hasselbring, B. M., Page, C. A., Sheppard, E. S. \& Krause, D. C. (2006). Transposon mutagenesis identifies genes associated with Mycoplasma pneumoniae gliding motility. J Bacteriol 188, 6335-6345.

Hegermann, J., Herrmann, R. \& Mayer, F. (2002). Cytoskeletal elements in the bacterium Mycoplasma pneumoniae. Naturwissenschaften 89, 453-458.

Henderson, G. P. \& Jensen, G. J. (2006). Three-dimensional structure of Mycoplasma pneumoniae's attachment organelle and a model for its role in gliding motility. Mol Microbiol 60, 376-385.

Himmelreich, R. (1997). Gesamtanalyse des Genoms von Mycoplasma pneumoniae. Thesis, University of Heidelberg.

Himmelreich, R., Hilbert, H., Plagens, H., Pirkl, E., Li, B. C. \& Herrmann, R. (1996). Complete sequence analysis of the genome of the bacterium Mycoplasma pneumoniae. Nucleic Acids Res 24, 4420-4429.

Hirano, T. (2005). Dynamic molecular linkers of the genome: the first decade of SMC proteins. Genes Dev 19, 1269-1287.

Hoppert, M. (2003). Microscopic Techniques in Biotechnology. Weinheim: Wiley-VCH.

Hu, P. C., Cole, R. M., Huabg, Y. S., Graham, J. A., Gardner, D. E., Collier, A. M. \& Clyde, W. A. (1982). Mycoplasma pneumoniae infection: role of a surface protein in the attachment organelle. Science 216, 313-315.

Kanehisa, M., Goto, S., Hattori, M., Aoki-Kinoshita, K. F., Itoh, M., Kawashima, S., Katayama, T., Araki, M. \& Hirakawa, M. (2006). From genomics to chemical genomics: new developments in KEGG. Nucleic Acids Res 34, D354-D357.

Krause, D. C. \& Balish, M. F. (2004). Cellular engineering in a minimal microbe: structure and assembly of the terminal organelle of Mycoplasma pneumoniae. Mol Microbiol 51, 917-924.

Krause, D. C., Leith, D. K., Wilson, R. M. \& Baseman, J. B. (1982). Identification of Mycoplasma pneumoniae proteins associated with hemadsorption and virulence. Infect Immun 35, 809-817. 
Meng, K. E. \& Pfister, R. M. (1980). Intracellular structures of Mycoplasma pneumoniae revealed after membrane removal. J Bacteriol 144, 390-399.

Neyrolles, O., Brenner, C., Prevost, M. C., Fontaine, C., Montagnier, L. \& Blanchard, A. (1998). Identification of two glycosylated components of Mycoplasma penetrans: a surface-exposed capsular polysaccharide and a glycolipid fraction. Microbiology 144, 1247-1255.

Nickel, W. (2003). The mystery of nonclassical protein secretion. A current view on cargo proteins and potential export routes. Eur J Biochem 270, 2109-2119.

Proft, T. \& Herrmann, R. (1994). Identification and characterization of hitherto unknown Mycoplasma pneumoniae proteins. Mol Microbiol 13, 337-348.

Puig, O., Caspary, F., Rigaut, G., Rutz, B., Bouveret, E., BragadoNilsson, E., Wilm, M. \& Seraphin, B. (2001). The tandem affinity purification (TAP) method: a general procedure of protein complex purification. Methods 24, 218-229.

Regula, J. T. (1999). Auf dem Weg zum Proteom von Mycoplasma pneumoniae. Thesis, University of Heidelberg.

Regula, J. T., Ueberle, B., Boguth, G., Görg, A., Schnölzer, M., Herrmann, R. \& Frank, R. (2000). Towards a two-dimensional proteome map of Mycoplasma pneumoniae. Electrophoresis 21, 3765-3780.

Regula, J. T., Boguth, G., Görg, A., Hegermann, J., Mayer, F., Frank, R. \& Herrmann, R. (2001). Defining the mycoplasma 'cytoskeleton': the protein composition of the Triton X-100 insoluble fraction of the bacterium Mycoplasma pneumoniae determined by 2-D gel electrophoresis and mass spectrometry. Microbiology 147, 1045-1057.

Rosengarten, R., Kirchhoff, H., Kerlen, G. \& Seack, K. H. (1988). The surface layer of Mycoplasma mobile $163 \mathrm{~K}$ and its possible relevance to cell cohesion and group motility. J Gen Microbiol 134, 275-281.

Salwinski, L., Miller, C. S., Smith, A. J., Pettit, F. K., Bowie, J. U. \& Eisenberg, D. (2004). The database of interacting proteins: 2004 update. Nucleic Acids Res 32, D449-D451.

Seybert, A., Herrmann, R. \& Frangakis, A. S. (2006). Structural analysis of Mycoplasma pneumoniae by cryo-electron tomography. J Struct Biol 156, 342-345.

Su, H.-C., Hutchison, C. A., III \& Giddings, M. C. (2007). Mapping phosphoproteins in Mycoplasma genitalium and Mycoplasma pneumoniae. BMC Microbiol 7, 63.

Waites, K. B. \& Talkington, D. F. (2004). Mycoplasma pneumoniae and its role as a human pathogen. Clin Microbiol Rev 17, 697-728.

Wenzel, R. \& Herrmann, R. (1989). Cloning of the complete Mycoplasma pneumoniae genome. Nucleic Acids Res 17, 7029-7040.

Wilson, M. H. \& Collier, A. M. (1976). Ultrastructural study of Mycoplasma pneumoniae in organ culture. J Bacteriol 125, 332-339.

Edited by: C. Citti 